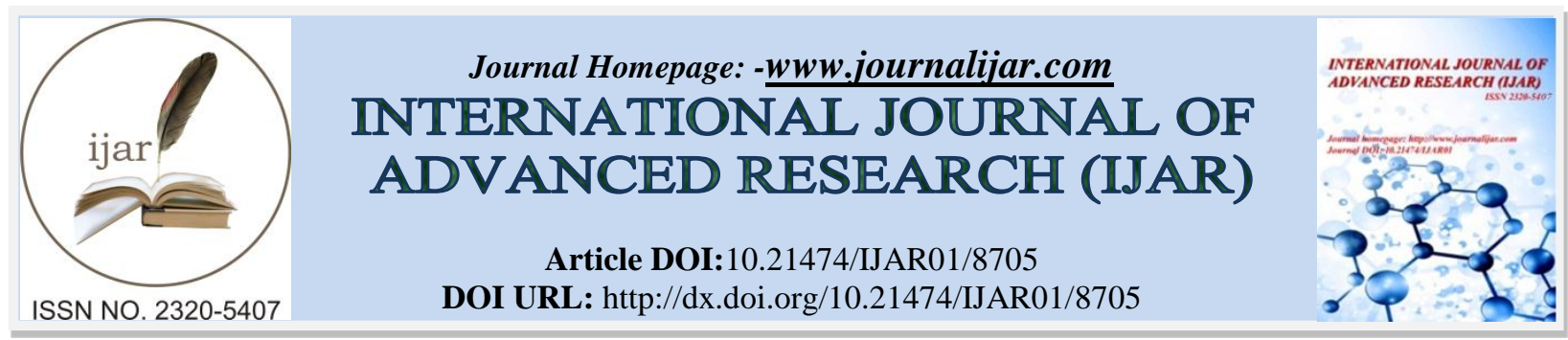

RESEARCH ARTICLE

\title{
AN EMPERICAL STUDY OF DEMONETIZATION \& ITS IMPACT ON RETHINKING OF TAX DISCLOSURE - A STUDY BASED ON NATIONAL CAPITAL REGION.
}

\author{
Ms. Suruchi Nain and Ms. Preeti Panse.
}

Assistant professor, School of commerce \& management Lingaya's university, Faridabad.

\section{Manuscript Info}

\section{Manuscript History}

Received: 16 January 2019

Final Accepted: 18 February 2019

Published: March 2019

Key words:-

Demonetization, Legal Tender, Black Money, Fake Currency.

\begin{abstract}
Demonetization refers to the withdrawal of particular currency (legal tender currency of country) from circulation. Demonetization done to reduce the black money, eradicate fake currency and to promote digital India. This is the bold step taken by the Indian government on 8 November 2016 for the betterment of the economy and country. In this paper, perception of general public toward tax disclosure after demonetization is analyzed through primary data collected .Questionnaire is prepared for primary data and filled with 81 respondents i.e. trader, manufacturer and service provider of Delhi NCR and this data used for further analyses by using descriptive table . After this research work, I concluded that almost all have neutral opinion toward demonetization impact on tax disclosure.
\end{abstract}

Copy Right, IJAR, 2019,. All rights reserved.

\section{Introduction:-}

Demonetization is an act of cancelling the legal tender status of a currency unit. The old unit of currency must be retired and replaced with a new currency unit .Legal Tender is any official medium of payment recognized by law that can be used to pay public or private debt, or to meet a financial obligation.

Prime Minister of India "Narendra Modi" announced the demonetization of higher currency notes in a spontaneous live televised address at 20:00 Indian Standard Time (IST) on 8 November 2016. In the announcement, Prime Minister Mr. Modi confirmed that the use of all 500 and 1000 banknotes of the Mahatma Gandhi Series would be worthless past midnight. All notes in lower denomination of Rs 100, Rs 50, Rs 20, Rs 10, Rs 5, Rs 2 and Re 1 and all coins continued to be valid and announced that new 500 and 2000 banknotes of the Mahatma Gandhi New Series will be issued in exchange for the old banknotes.

The reasoning given by Prime Minister Mr. Modi was:-

1. To Deal with the Black Money in the economy.

2. To lower the Cash circulation in the country which "is directly related to corruption in our country," according to PM Modi.

3. To eradicate fake currency which have been used by terror groups to fund terrorism in India.

4. To promote 'payments through cards and digital means ', 'discourage transactions in cash', and 'shift the payments from cash-dominated to non-cash/less cash payments' and make "cashless economy" (digital India).

Corresponding Author:-Suruchi Nain.

Address:-Assistant professor, School of commerce \& management Lingaya's university, Faridabad. 
As Cash is an insignificant component of Black Asset and Cash recovery has been only 6\% of the undisclosed income seized from income tax evaders.

Black Money refers to the sum person own which is unaccounted. It includes basically money which is earned through any illegal activity controlled by country regulations. People generally received in cash and hide this money and hence not taxed.

Government introduced income declaration scheme, kept deadline for same and promised to keep the data confidential .Penalties were different for declared income, not declared and detected in search and seize cases. The money from the scheme would be used for projects in irrigation, housing, toilets, infrastructure, primary education, primary health and livelihood so that there is justice and equality, said the Statement of Objects and Reasons of the Bill.

The sudden move to demonetization of currency notes is not new.1,000 and higher denomination notes were demonetized earlier also in 1946 and in 1978. However, this is the first time that 2,000 currency note is being introduced.

\section{Need and Objective of the study.}

As in Current Scenario in India, before demonetization, some persons were holing huge amount of hard cash at their home, they don't disclose correct amount of taxable income in their Income Tax Return,black money was in high proportion in India. Some persons were doing payment through cash for which have no records and they don't need to show it in their Income Tax Return, and by doing so they were saving tax. So as to lower the cash transaction, tackle black money, eliminate fake currency, demonetization was the urgent need of the country so that correct disclosure can be made in. Various laws implemented by Prime Minister Narendra Modi to disclose the unaccounted money and budget have also various new implications for fair transaction and disclosure of income. Payment banking has increased after demonetization due to Digital India move led by PM Modi. One page income tax return also a great move for easiness and correct disclosure of taxable amount. So, the study was needed to analyze the perception of public toward tax disclosure after demonetization.

\section{Review of Literature}

(kaur, 2016) apprised in her study that demonetization refers to Withdrawal of a particular form of currency from circulation. This move would be positive for sectors like Banking and Infrastructure in the medium term according to equity market perspective and could be negative for sectors like Consumer Durables, Luxury items, Gems and Jewellery, Real Estate and allied sectors, in the near to medium term. It can lead to improved tax compliance, better fiscal balance, lower inflation, lower corruption, complete elimination of fake currency and another stepping stone for sustained economic growth in the longer term.

(Chanda, 2016)mentioned in her study that in economic reforms, there are costs that are immediate, obvious, and asymmetrical while the gains are diffuse and often hard to measure. Modi has staked his position as a leader capable of making bold decisions as demonetization and unprecedented boldness of this decision itself seems, at least in urban and semi-urban, India to have played in his favor. This support is not unconditional and it depends on the speed with which the current liquidity crunch is resolved, and also how well the battle is played out in the media. While Modi may own the narrative now, much of the success depends on the next steps that his government introduces to tackle black money as demonetization would be the first step in this.

(Devos \& Zackrisson, 2015) apprised in his study that combination of both influential and enforcement measures have been applied by governments to deal with tax non-compliance. With growing pressure to raise revenue in the current economic climate, governments need to assess the efficiency of various compliance measures. Strategies adopted by tax authorities globally were analyzed specifically in Australia and Norway, regarding the public disclosure of tax information and its likely compliance impact. It provides an imminent about this in the setting of one country, while on other hand some limited disclosure may supplement other compliance strategies in other.

\section{Research Methodology:-}

\section{Population of the study:}

Population of the study consisted of the manufacturer, trader and service provider of Delhi NCR only. 


\section{Research Design:}

Descriptive Study

\section{Sample size:}

Sample size of the study was 81 respondents.

\section{Sampling technique:}

Cluster and Snow Ball Sampling used.

Data collection tools:

This research was basically based on primary data collected using a structured questionnaire administered to 81 respondents.

\section{Data analysis tool:}

Collected data was analyzed using SPSS software by using statistical tool descriptive statistics.

\section{Analysis and Interpretation}

Reliability Test

Cronbach's Alpha

0.888

\section{No. Of Items}

23.

Cronbach's Alpha Value is more than 0.70 Which Shows sufficient reliability of responses collected through Questionnaire.

\begin{tabular}{|c|c|c|c|c|c|c|}
\hline \multicolumn{7}{|l|}{ ANOVA } \\
\hline & & $\begin{array}{ll}\text { Sum } \\
\text { Squares }\end{array}$ & $\mathrm{df}$ & $\begin{array}{l}\text { Mean } \\
\text { Square }\end{array}$ & $\mathrm{F}$ & Sig. \\
\hline \multirow{3}{*}{$\begin{array}{l}\text { [If economy goes } \\
\text { cashless, rent received } \\
\text { through accounts will be } \\
\text { disclosed in Income Tax } \\
\text { Return.] }\end{array}$} & $\begin{array}{l}\text { Between } \\
\text { Groups }\end{array}$ & 13.556 & 2 & 6.778 & 5.931 & .004 \\
\hline & Within Groups & 89.136 & 78 & 1.143 & & \\
\hline & Total & 102.691 & 80 & & & \\
\hline \multirow{3}{*}{$\begin{array}{l}\text { [filling Tax Return and } \\
\text { understanding it is } \\
\text { Difficult] }\end{array}$} & $\begin{array}{l}\text { Between } \\
\text { Groups }\end{array}$ & 4.030 & 2 & 2.015 & 2.465 & .092 \\
\hline & Within Groups & 62.957 & 77 & .818 & & \\
\hline & Total & 66.988 & 79 & & & \\
\hline \multirow{3}{*}{$\begin{array}{l}\text { Do you have your } \\
\text { business registered in } \\
\text { any applicable law? }\end{array}$} & $\begin{array}{l}\text { Between } \\
\text { Groups }\end{array}$ & 1.461 & 2 & .731 & 3.058 & .053 \\
\hline & Within Groups & 18.637 & 78 & .239 & & \\
\hline & Total & 20.099 & 80 & & & \\
\hline \multirow[t]{3}{*}{$\begin{array}{l}\text { Do you disclose your all } \\
\text { sources of income? }\end{array}$} & $\begin{array}{l}\text { Between } \\
\text { Groups }\end{array}$ & .328 & 2 & .164 & .712 & .494 \\
\hline & Within Groups & 17.993 & 78 & .231 & & \\
\hline & Total & 18.321 & 80 & & & \\
\hline \multirow[t]{3}{*}{ Do you have PAN? } & $\begin{array}{l}\text { Between } \\
\text { Groups }\end{array}$ & .039 & 2 & .020 & .276 & .759 \\
\hline & Within Groups & 5.516 & 78 & .071 & & \\
\hline & Total & 5.556 & 80 & & & \\
\hline \multirow{3}{*}{$\begin{array}{l}\text { Do you file your income } \\
\text { tax return on regular } \\
\text { basis? }\end{array}$} & $\begin{array}{l}\text { Between } \\
\text { Groups } \\
\end{array}$ & .089 & 2 & .044 & .240 & .787 \\
\hline & Within Groups & 14.454 & 78 & .185 & & \\
\hline & Total & 14.543 & 80 & & & \\
\hline $\begin{array}{l}\text { [Filling of return by } \\
\text { CA, CS and other }\end{array}$ & $\begin{array}{l}\text { Between } \\
\text { Groups }\end{array}$ & .952 & 2 & .476 & .554 & .577 \\
\hline
\end{tabular}




\begin{tabular}{|c|c|c|c|c|c|c|}
\hline \multirow{2}{*}{$\begin{array}{l}\text { professional person is } \\
\text { Necessary.] }\end{array}$} & Within Groups & 67.048 & 78 & .860 & & \\
\hline & Total & 68.000 & 80 & & & \\
\hline \multirow{3}{*}{$\begin{array}{l}\text { Do you disclose actual } \\
\text { Cash in hand and Bank } \\
\text { balance in Income Tax } \\
\text { Return? }\end{array}$} & $\begin{array}{l}\text { Between } \\
\text { Groups }\end{array}$ & 1.753 & 2 & .877 & 3.991 & .022 \\
\hline & Within Groups & 17.136 & 78 & .220 & & \\
\hline & Total & 18.889 & 80 & & & \\
\hline \multirow[t]{3}{*}{$\begin{array}{l}\text { Do you fill ITR } 4 \\
\text { (which is of } 24 \text { pages)? }\end{array}$} & $\begin{array}{l}\text { Between } \\
\text { Groups }\end{array}$ & 1.109 & 2 & .554 & 2.262 & .111 \\
\hline & Within Groups & 19.114 & 78 & .245 & & \\
\hline & Total & 20.222 & 80 & & & \\
\hline \multirow{3}{*}{$\begin{array}{l}\text { Did you avail any } \\
\text { scheme of undisclosed } \\
\text { income issued by the } \\
\text { government? }\end{array}$} & $\begin{array}{l}\text { Between } \\
\text { Groups }\end{array}$ & .089 & 2 & .044 & .240 & .787 \\
\hline & Within Groups & 14.454 & 78 & .185 & & \\
\hline & Total & 14.543 & 80 & & & \\
\hline \multirow{3}{*}{$\begin{array}{l}\text { [Taxes have not been } \\
\text { paid will now be } \\
\text { recovered due to } \\
\text { demonetization.] }\end{array}$} & $\begin{array}{l}\text { Between } \\
\text { Groups }\end{array}$ & 3.615 & 2 & 1.808 & 1.781 & .175 \\
\hline & Within Groups & 79.150 & 78 & 1.015 & & \\
\hline & Total & 82.765 & 80 & & & \\
\hline \multirow{3}{*}{$\begin{array}{l}\text { [Due to demonetization, } \\
\text { inflation rate of } \\
\text { economy decreases.] }\end{array}$} & $\begin{array}{l}\text { Between } \\
\text { Groups }\end{array}$ & 3.771 & 2 & 1.885 & 1.568 & .215 \\
\hline & Within Groups & 93.810 & 78 & 1.203 & & \\
\hline & Total & 97.580 & 80 & & & \\
\hline \multirow{3}{*}{$\begin{array}{l}\text { [People who were } \\
\text { holding black money in } \\
\text { cash has not been able to } \\
\text { exchange much as they } \\
\text { would be in a fear of } \\
\text { getting prosecuted by } \\
\text { the authorities.] }\end{array}$} & $\begin{array}{l}\text { Between } \\
\text { Groups }\end{array}$ & 7.412 & 2 & 3.706 & 3.070 & .052 \\
\hline & Within Groups & 94.168 & 78 & 1.207 & & \\
\hline & Total & 101.580 & 80 & & & \\
\hline \multirow{3}{*}{$\begin{array}{l}\text { [There is increase in } \\
\text { return income of persons } \\
\text { compared to pre- } \\
\text { demonetized return } \\
\text { income.] }\end{array}$} & $\begin{array}{l}\text { Between } \\
\text { Groups }\end{array}$ & 2.794 & 2 & 1.397 & 1.665 & .196 \\
\hline & Within Groups & 65.429 & 78 & .839 & & \\
\hline & Total & 68.222 & 80 & & & \\
\hline \multirow{3}{*}{$\begin{array}{l}\text { [Disclosure of all } \\
\text { business transactions } \\
\text { will increase lead to fair } \\
\text { return after } \\
\text { demonetization.] }\end{array}$} & $\begin{array}{l}\text { Between } \\
\text { Groups }\end{array}$ & 11.832 & 2 & 5.916 & 6.789 & .002 \\
\hline & Within Groups & 67.971 & 78 & .871 & & \\
\hline & Total & 79.802 & 80 & & & \\
\hline \multirow{3}{*}{$\begin{array}{l}\text { [Demonetization drive } \\
\text { is a good development } \\
\text { for payment banks } \\
\text { which will lead to } \\
\text { Cashless Economy.] }\end{array}$} & $\begin{array}{l}\text { Between } \\
\text { Groups }\end{array}$ & 20.024 & 2 & 10.012 & 8.658 & .000 \\
\hline & Within Groups & 90.198 & 78 & 1.156 & & \\
\hline & Total & 110.222 & 80 & & & \\
\hline \multirow{3}{*}{$\begin{array}{l}\text { [Complete disclosure of } \\
\text { Balances and Account } \\
\text { details can reduce } \\
\text { complication of non- } \\
\text { disclosure.] }\end{array}$} & $\begin{array}{l}\text { Between } \\
\text { Groups }\end{array}$ & 3.087 & 2 & 1.543 & 2.036 & .137 \\
\hline & Within Groups & 59.136 & 78 & .758 & & \\
\hline & Total & 62.222 & 80 & & & \\
\hline \multirow{3}{*}{$\begin{array}{l}\text { [No transaction above } 3 \\
\text { lakh will be allowed in } \\
\text { Cash. This move will } \\
\text { lead to more transparent } \\
\text { income disclosure.] }\end{array}$} & $\begin{array}{l}\text { Between } \\
\text { Groups }\end{array}$ & 4.787 & 2 & 2.394 & 2.315 & .105 \\
\hline & Within Groups & 80.645 & 78 & 1.034 & & \\
\hline & Total & 85.432 & 80 & & & \\
\hline [After demonetization & Between & 6.447 & 2 & 3.223 & 3.615 & .032 \\
\hline
\end{tabular}




\begin{tabular}{|c|c|c|c|c|c|c|}
\hline \multirow{3}{*}{$\begin{array}{l}\text { drive, disclosure of } \\
\text { correct and complete } \\
\text { information of Cash in } \\
\text { hand and Bank balance } \\
\text { will increase.] }\end{array}$} & Groups & & & & & \\
\hline & Within Groups & 69.553 & 78 & .892 & & \\
\hline & Total & 76.000 & 80 & & & \\
\hline \multirow{3}{*}{$\begin{array}{l}\text { [Proposed One page } \\
\text { Income Tax Return } \\
\text { Form for having income } \\
\text { less than 5 lakh in } \\
\text { previous year will make } \\
\text { the system easy for } \\
\text { disclosure.] }\end{array}$} & $\begin{array}{l}\text { Between } \\
\text { Groups }\end{array}$ & 2.295 & 2 & 1.148 & 1.245 & .294 \\
\hline & Within Groups & 71.927 & 78 & .922 & & \\
\hline & Total & 74.222 & 80 & & & \\
\hline \multirow{3}{*}{$\begin{array}{l}\text { [Presumptive Tax for } \\
\text { small traders now } 6 \% \\
\text { instead of } 8 \% \text { for full } \\
\text { 1ncash turnover. This } \\
\text { will increase tax } \\
\text { disclosure of small } \\
\text { traders.] }\end{array}$} & $\begin{array}{l}\text { Between } \\
\text { Groups }\end{array}$ & .251 & 2 & .125 & .151 & .860 \\
\hline & Within Groups & 63.949 & 77 & .831 & & \\
\hline & Total & 64.200 & 79 & & & \\
\hline \multirow{3}{*}{$\begin{array}{l}\text { [In family, now more } \\
\text { adult group will indulge } \\
\text { in filing Income Tax } \\
\text { Return after } \\
\text { demonetization as } \\
\text { compared to before.] }\end{array}$} & $\begin{array}{l}\text { Between } \\
\text { Groups }\end{array}$ & 1.330 & 2 & .665 & .747 & .477 \\
\hline & Within Groups & 69.436 & 78 & .890 & & \\
\hline & Total & 70.765 & 80 & & & \\
\hline \multirow{3}{*}{$\begin{array}{l}\text { [People will make use } \\
\text { of HUF after } \\
\text { demonetization which } \\
\text { will lead to more tax } \\
\text { disclosure.] }\end{array}$} & $\begin{array}{l}\text { Between } \\
\text { Groups }\end{array}$ & 1.915 & 2 & .957 & 1.326 & .271 \\
\hline & Within Groups & 56.308 & 78 & .722 & & \\
\hline & Total & 58.222 & 80 & & & \\
\hline
\end{tabular}

\begin{tabular}{|c|c|c|c|c|c|c|c|}
\hline \multirow{2}{*}{\multicolumn{8}{|c|}{$\begin{array}{l}\text { Multiple Comparisons } \\
\text { TUKFYHSD }\end{array}$}} \\
\hline & & & & & & & \\
\hline \multirow[t]{2}{*}{$\begin{array}{l}\text { Dependent } \\
\text { Variable }\end{array}$} & \multirow{2}{*}{$\begin{array}{l}\text { (I) What is } \\
\text { your source } \\
\text { of income? }\end{array}$} & \multirow{2}{*}{$\begin{array}{l}(\mathrm{J}) \text { What is } \\
\text { your source } \\
\text { of income? }\end{array}$} & \multirow{2}{*}{$\begin{array}{l}\text { Mean } \\
\text { Difference } \\
\text { (I-J) }\end{array}$} & \multirow[t]{2}{*}{$\begin{array}{l}\text { Std. } \\
\text { Error }\end{array}$} & \multirow[t]{2}{*}{ Sig. } & \multicolumn{2}{|l|}{$\begin{array}{l}95 \% \\
\text { Interval }\end{array}$} \\
\hline & & & & & & $\begin{array}{l}\text { Lower } \\
\text { Bound }\end{array}$ & $\begin{array}{l}\text { Upper } \\
\text { Bound }\end{array}$ \\
\hline \multirow{6}{*}{$\begin{array}{l}\text { Do you have } \\
\text { your business } \\
\text { registered in any } \\
\text { applicable law? }\end{array}$} & \multirow[t]{2}{*}{ Manufacturer } & $\begin{array}{l}\text { Service } \\
\text { Provider }\end{array}$ & $.326^{*}$ & .132 & .042 & .01 & .64 \\
\hline & & Trader & .238 & .151 & .261 & -.12 & .60 \\
\hline & \multirow{2}{*}{$\begin{array}{l}\text { Service } \\
\text { Provider }\end{array}$} & Manufacturer & $-.326^{*}$ & .132 & .042 & -.64 & -.01 \\
\hline & & Trader & -.088 & .132 & .785 & -.40 & .23 \\
\hline & \multirow[t]{2}{*}{ Trader } & Manufacturer & -.238 & .151 & .261 & -.60 & .12 \\
\hline & & $\begin{array}{l}\text { Service } \\
\text { Provider }\end{array}$ & .088 & .132 & .785 & -.23 & .40 \\
\hline \multirow{6}{*}{$\begin{array}{l}\text { [If economy } \\
\text { goes cashless, } \\
\text { rent received } \\
\text { through accounts } \\
\text { will be disclosed } \\
\text { in Income Tax } \\
\text { Return.] }\end{array}$} & \multirow[t]{2}{*}{ Manufacturer } & $\begin{array}{l}\text { Service } \\
\text { Provider }\end{array}$ & -.330 & .289 & .493 & -1.02 & .36 \\
\hline & & Trader & .667 & .330 & .114 & -.12 & 1.45 \\
\hline & \multirow{2}{*}{$\begin{array}{l}\text { Service } \\
\text { Provider }\end{array}$} & Manufacturer & .330 & .289 & .493 & -.36 & 1.02 \\
\hline & & Trader & $.996^{*}$ & .289 & .003 & .31 & 1.69 \\
\hline & \multirow[t]{2}{*}{ Trader } & Manufacturer & -.667 & .330 & .114 & -1.45 & .12 \\
\hline & & $\begin{array}{l}\text { Service } \\
\text { Provider }\end{array}$ & $-.996^{*}$ & .289 & .003 & -1.69 & -.31 \\
\hline Do you disclose & Manufacturer & Service & -.099 & .130 & .728 & -.41 & .21 \\
\hline
\end{tabular}




\begin{tabular}{|c|c|c|c|c|c|c|c|}
\hline \multirow{6}{*}{$\begin{array}{l}\text { your all sources } \\
\text { of income? }\end{array}$} & & Provider & & & & & \\
\hline & & Trader & .048 & .148 & .945 & -.31 & .40 \\
\hline & \multirow{2}{*}{$\begin{array}{l}\text { Service } \\
\text { Provider }\end{array}$} & Manufacturer & .099 & .130 & .728 & -.21 & .41 \\
\hline & & Trader & .147 & .130 & .500 & -.16 & .46 \\
\hline & \multirow[t]{2}{*}{ Trader } & Manufacturer & -.048 & .148 & .945 & -.40 & .31 \\
\hline & & $\begin{array}{l}\text { Service } \\
\text { Provider }\end{array}$ & -.147 & .130 & .500 & -.46 & .16 \\
\hline \multirow[t]{6}{*}{$\begin{array}{l}\text { Do you have } \\
\text { PAN? }\end{array}$} & \multirow[t]{2}{*}{ Manufacturer } & $\begin{array}{l}\text { Service } \\
\text { Provider }\end{array}$ & -.044 & .072 & .815 & -.22 & .13 \\
\hline & & Trader & .000 & .082 & 1.000 & -.20 & .20 \\
\hline & \multirow{2}{*}{$\begin{array}{l}\text { Service } \\
\text { Provider }\end{array}$} & Manufacturer & .044 & .072 & .815 & -.13 & .22 \\
\hline & & Trader & .044 & .072 & .815 & -.13 & .22 \\
\hline & \multirow[t]{2}{*}{ Trader } & Manufacturer & .000 & .082 & 1.000 & -.20 & .20 \\
\hline & & $\begin{array}{l}\text { Service } \\
\text { Provider }\end{array}$ & -.044 & .072 & .815 & -.22 & .13 \\
\hline \multirow{6}{*}{$\begin{array}{l}\text { Do you file your } \\
\text { income tax } \\
\text { return on regular } \\
\text { basis? }\end{array}$} & \multirow[t]{2}{*}{ Manufacturer } & $\begin{array}{l}\text { Service } \\
\text { Provider }\end{array}$ & -.033 & 117 & 957 & -.31 & .25 \\
\hline & & Trader & .048 & .133 & .932 & -.27 & .37 \\
\hline & \multirow{2}{*}{$\begin{array}{l}\text { Service } \\
\text { Provider }\end{array}$} & Manufacturer & .033 & .117 & .957 & -.25 & .31 \\
\hline & & Trader & .081 & .117 & .769 & -.20 & .36 \\
\hline & \multirow[t]{2}{*}{ Trader } & Manufacturer & -.048 & .133 & .932 & -.37 & .27 \\
\hline & & $\begin{array}{l}\text { Service } \\
\text { Provider }\end{array}$ & -.081 & 117 & .769 & -.36 & .20 \\
\hline \multirow{6}{*}{$\begin{array}{l}\text { [filling Tax } \\
\text { Return and } \\
\text { understanding it } \\
\text { is Difficult] }\end{array}$} & \multirow[t]{2}{*}{ Manufacturer } & $\begin{array}{l}\text { Service } \\
\text { Provider }\end{array}$ & -.509 & 246 & .103 & -1.10 & .08 \\
\hline & & Trader & -.143 & .279 & .866 & -.81 & .52 \\
\hline & \multirow{2}{*}{$\begin{array}{l}\text { Service } \\
\text { Provider }\end{array}$} & Manufacturer & .509 & .246 & .103 & -.08 & 1.10 \\
\hline & & Trader & .366 & .246 & .302 & -.22 & .95 \\
\hline & \multirow[t]{2}{*}{ Trader } & Manufacturer & .143 & .279 & .866 & -.52 & .81 \\
\hline & & $\begin{array}{l}\text { Service } \\
\text { Provider }\end{array}$ & -.366 & .246 & .302 & -.95 & .22 \\
\hline \multirow{6}{*}{\begin{tabular}{l}
\multicolumn{2}{l}{ [Filling of return } \\
by CA, CS and \\
other \\
professional \\
person \\
Necessary.] is
\end{tabular}} & \multirow[t]{2}{*}{ Manufacturer } & $\begin{array}{l}\text { Service } \\
\text { Provider }\end{array}$ & -.238 & .251 & 611 & -.84 & .36 \\
\hline & & Trader & -.048 & .286 & .985 & -.73 & .64 \\
\hline & \multirow{2}{*}{$\begin{array}{l}\text { Service } \\
\text { Provider }\end{array}$} & Manufacturer & .238 & .251 & .611 & -.36 & .84 \\
\hline & & Trader & .190 & .251 & .729 & -.41 & .79 \\
\hline & \multirow[t]{2}{*}{ Trader } & Manufacturer & .048 & .286 & .985 & -.64 & .73 \\
\hline & & $\begin{array}{l}\text { Service } \\
\text { Provider }\end{array}$ & -.190 & .251 & .729 & -.79 & 41 \\
\hline \multirow{6}{*}{$\begin{array}{lr}\text { Do you disclose } \\
\text { actual Cash in } \\
\text { hand and Bank } \\
\text { balance } \\
\text { Income } \\
\text { Return? }\end{array}$} & \multirow[t]{2}{*}{ Manufacturer } & $\begin{array}{l}\text { Service } \\
\text { Provider }\end{array}$ & -.004 & .127 & 1.000 & -.31 & .30 \\
\hline & & Trader & .333 & .145 & .061 & -.01 & .68 \\
\hline & \multirow{2}{*}{$\begin{array}{l}\text { Service } \\
\text { Provider }\end{array}$} & Manufacturer & .004 & .127 & 1.000 & -.30 & .31 \\
\hline & & Trader & $.337^{*}$ & .127 & .026 & .03 & .64 \\
\hline & \multirow[t]{2}{*}{ Trader } & Manufacturer & -.333 & .145 & .061 & -.68 & .01 \\
\hline & & $\begin{array}{l}\text { Service } \\
\text { Provider }\end{array}$ & $-.337^{*}$ & .127 & .026 & -.64 & -.03 \\
\hline \multirow{6}{*}{$\begin{array}{l}\text { Do you fill ITR } 4 \\
\text { (which is of } 24 \\
\text { pages)? }\end{array}$} & Manufacturer & $\begin{array}{l}\text { Service } \\
\text { Provider }\end{array}$ & .278 & .134 & .101 & -.04 & .60 \\
\hline & & Trader & .238 & .153 & .270 & -.13 & .60 \\
\hline & Service & Manufacturer & -.278 & .134 & .101 & -.60 & .04 \\
\hline & Provider & Trader & -.040 & .134 & .951 & -.36 & .28 \\
\hline & Trader & Manufacturer & -.238 & .153 & .270 & -.60 & .13 \\
\hline & & Service & .040 & .134 & .951 & -.28 & .36 \\
\hline
\end{tabular}




\begin{tabular}{|c|c|c|c|c|c|c|c|}
\hline & & Provider & & & & & \\
\hline \multirow{6}{*}{$\begin{array}{l}\text { Did you avail } \\
\text { any scheme of } \\
\text { undisclosed } \\
\text { income issued by } \\
\text { the government? }\end{array}$} & \multirow[t]{2}{*}{ Manufacturer } & $\begin{array}{l}\text { Service } \\
\text { Provider }\end{array}$ & .081 & .117 & .769 & -.20 & .36 \\
\hline & & Trader & .048 & .133 & .932 & -.27 & .37 \\
\hline & \multirow{2}{*}{$\begin{array}{l}\text { Service } \\
\text { Provider }\end{array}$} & Manufacturer & -.081 & .117 & .769 & -.36 & .20 \\
\hline & & Trader & -.033 & .117 & .957 & -.31 & .25 \\
\hline & \multirow[t]{2}{*}{ Trader } & Manufacturer & $\begin{array}{l}-.048 \\
\end{array}$ & .133 & .932 & -.37 & .27 \\
\hline & & $\begin{array}{l}\text { Service } \\
\text { Provider }\end{array}$ & .033 & .117 & .957 & -.25 & .31 \\
\hline \multirow{6}{*}{$\begin{array}{l}\text { [Taxes have not } \\
\text { been paid will } \\
\text { now be } \\
\text { recovered due to } \\
\text { demonetization.] }\end{array}$} & \multirow[t]{2}{*}{ Manufacturer } & $\begin{array}{l}\text { Service } \\
\text { Provider }\end{array}$ & -.267 & .273 & .591 & -.92 & .38 \\
\hline & & Trader & .238 & .311 & .725 & -.50 & .98 \\
\hline & \multirow{2}{*}{$\begin{array}{l}\text { Service } \\
\text { Provider }\end{array}$} & Manufacturer & .267 & .273 & .591 & -.38 & .92 \\
\hline & & Trader & .505 & .273 & .159 & -.15 & 1.16 \\
\hline & \multirow[t]{2}{*}{ Trader } & Manufacturer & -.238 & .311 & .725 & -.98 & .50 \\
\hline & & $\begin{array}{l}\text { Service } \\
\text { Provider }\end{array}$ & -.505 & .273 & 159 & -1.16 & .15 \\
\hline \multirow{6}{*}{$\begin{array}{l}\text { [Due to } \\
\text { demonetization, } \\
\text { inflation rate of } \\
\text { economy } \\
\text { decreases.] }\end{array}$} & \multirow[t]{2}{*}{ Manufacturer } & $\begin{array}{l}\text { Service } \\
\text { Provider }\end{array}$ & -.143 & .297 & .880 & -.85 & .57 \\
\hline & & Trader & .381 & .338 & .501 & -.43 & 1.19 \\
\hline & \multirow{2}{*}{$\begin{array}{l}\text { Service } \\
\text { Provider }\end{array}$} & Manufacturer & .143 & 297 & .880 & -.57 & .85 \\
\hline & & Trader & .524 & .297 & .188 & -.19 & 1.23 \\
\hline & \multirow[t]{2}{*}{ Trader } & Manufacturer & -.381 & .338 & .501 & -1.19 & .43 \\
\hline & & $\begin{array}{l}\text { Service } \\
\text { Provider }\end{array}$ & -.524 & .297 & .188 & -1.23 & .19 \\
\hline \multirow{6}{*}{$\begin{array}{l}\text { [People who } \\
\text { were holding } \\
\text { black money in } \\
\text { cash has not been } \\
\text { able to exchange } \\
\text { much as they } \\
\text { would be in a } \\
\text { fear of getting } \\
\text { prosecuted by } \\
\text { the authorities.] }\end{array}$} & \multirow[t]{2}{*}{ Manufacturer } & $\begin{array}{l}\text { Service } \\
\text { Provider }\end{array}$ & -.462 & .297 & .273 & -1.17 & .25 \\
\hline & & Trader & .238 & .339 & .763 & -.57 & 1.05 \\
\hline & \multirow{2}{*}{$\begin{array}{l}\text { Service } \\
\text { Provider }\end{array}$} & Manufacturer & .462 & .297 & .273 & -.25 & 1.17 \\
\hline & & Trader & .700 & .297 & .055 & -.01 & 1.41 \\
\hline & \multirow[t]{2}{*}{ Trader } & Manufacturer & -.238 & .339 & .763 & -1.05 & .57 \\
\hline & & $\begin{array}{l}\text { Service } \\
\text { Provider }\end{array}$ & -.700 & .297 & .055 & -1.41 & .01 \\
\hline \multirow{6}{*}{$\begin{array}{l}\text { [There is } \\
\text { increase in return } \\
\text { income of } \\
\text { persons } \\
\text { compared to pre- } \\
\text { demonetized } \\
\text { return income.] }\end{array}$} & \multirow[t]{2}{*}{ Manufacturer } & $\begin{array}{l}\text { Service } \\
\text { Provider }\end{array}$ & -.286 & .248 & .485 & -.88 & .31 \\
\hline & & Trader & .143 & .283 & .869 & -.53 & .82 \\
\hline & \multirow{2}{*}{$\begin{array}{l}\text { Service } \\
\text { Provider }\end{array}$} & Manufacturer & .286 & .248 & .485 & -.31 & .88 \\
\hline & & Trader & .429 & .248 & .201 & -.16 & 1.02 \\
\hline & \multirow[t]{2}{*}{ Trader } & Manufacturer & -.143 & .283 & .869 & -.82 & .53 \\
\hline & & $\begin{array}{l}\text { Service } \\
\text { Provider }\end{array}$ & -.429 & .248 & .201 & -1.02 & .16 \\
\hline \multirow{6}{*}{$\begin{array}{l}\text { [Disclosure of } \\
\text { all business } \\
\text { transactions will } \\
\text { increase lead to } \\
\text { fair return after } \\
\text { demonetization.] }\end{array}$} & \multirow[t]{2}{*}{ Manufacturer } & $\begin{array}{l}\text { Service } \\
\text { Provider }\end{array}$ & $-.626^{*}$ & .253 & .040 & -1.23 & -.02 \\
\hline & & Trader & .238 & .288 & .688 & -.45 & .93 \\
\hline & \multirow{2}{*}{$\begin{array}{l}\text { Service } \\
\text { Provider }\end{array}$} & Manufacturer & $.626^{*}$ & .253 & .040 & .02 & 1.23 \\
\hline & & Trader & $.864^{*}$ & .253 & .003 & .26 & 1.47 \\
\hline & \multirow[t]{2}{*}{ Trader } & Manufacturer & -.238 & .288 & .688 & -.93 & .45 \\
\hline & & $\begin{array}{l}\text { Service } \\
\text { Provider }\end{array}$ & $-.864^{*}$ & .253 & .003 & -1.47 & -.26 \\
\hline \multirow{2}{*}{$\begin{array}{l}\text { [Demonetization } \\
\text { drive is a good } \\
\text { development for }\end{array}$} & \multirow[t]{2}{*}{ Manufacturer } & $\begin{array}{l}\text { Service } \\
\text { Provider }\end{array}$ & -.495 & .291 & .212 & -1.19 & .20 \\
\hline & & Trader & .714 & .332 & .086 & -.08 & 1.51 \\
\hline
\end{tabular}




\begin{tabular}{|c|c|c|c|c|c|c|c|}
\hline \multirow{4}{*}{$\begin{array}{l}\text { payment banks } \\
\text { which will lead } \\
\text { to Cashless } \\
\text { Economy.] }\end{array}$} & \multirow{2}{*}{$\begin{array}{l}\text { Service } \\
\text { Provider }\end{array}$} & Manufacturer & .495 & .291 & .212 & -.20 & 1.19 \\
\hline & & Trader & $1.209^{*}$ & .291 & .000 & .51 & 1.90 \\
\hline & \multirow[t]{2}{*}{ Trader } & Manufacturer & -.714 & .332 & .086 & -1.51 & .08 \\
\hline & & $\begin{array}{l}\text { Service } \\
\text { Provider }\end{array}$ & -1.209 & .291 & .000 & -1.90 & -.51 \\
\hline \multirow{6}{*}{$\begin{array}{l}\text { [Complete } \\
\text { disclosure of } \\
\text { Balances and } \\
\text { Account details } \\
\text { can reduce } \\
\text { complication of } \\
\text { non-disclosure.] }\end{array}$} & \multirow[t]{2}{*}{ Manufacturer } & $\begin{array}{l}\text { Service } \\
\text { Provider }\end{array}$ & -.432 & 236 & 165 & -1.00 & .13 \\
\hline & & Trader & -.095 & .269 & .933 & -.74 & .55 \\
\hline & \multirow{2}{*}{$\begin{array}{l}\text { Service } \\
\text { Provider }\end{array}$} & Manufacturer & .432 & .236 & .165 & -.13 & 1.00 \\
\hline & & Trader & .337 & .236 & .331 & -.23 & .90 \\
\hline & \multirow{2}{*}{ Trader } & Manufacturer & .095 & .269 & .933 & -.55 & .74 \\
\hline & & $\begin{array}{l}\text { Service } \\
\text { Provider }\end{array}$ & -.337 & .236 & .331 & -.90 & .23 \\
\hline \multirow{6}{*}{$\begin{array}{l}\text { [No transaction } \\
\text { above } 3 \text { lakh will } \\
\text { be allowed in } \\
\text { Cash. This move } \\
\text { will lead to more } \\
\text { transparent } \\
\text { income } \\
\text { disclosure.] }\end{array}$} & \multirow[t]{2}{*}{ Manufacturer } & $\begin{array}{l}\text { Service } \\
\text { Provider }\end{array}$ & -.462 & .275 & .220 & -1.12 & .20 \\
\hline & & Trader & .048 & .314 & .987 & -.70 & .80 \\
\hline & \multirow{2}{*}{$\begin{array}{l}\text { Service } \\
\text { Provider }\end{array}$} & Manufacturer & .462 & .275 & .220 & -.20 & 1.12 \\
\hline & & Trader & .509 & .275 & .160 & -.15 & 1.17 \\
\hline & \multirow[t]{2}{*}{ Trader } & Manufacturer & -.048 & .314 & 987 & -.80 & .70 \\
\hline & & $\begin{array}{l}\text { Service } \\
\text { Provider }\end{array}$ & -.509 & .275 & 160 & -1.17 & .15 \\
\hline \multirow{6}{*}{$\begin{array}{l}\text { [After } \\
\text { demonetization } \\
\text { drive, disclosure } \\
\text { of correct and } \\
\text { complete } \\
\text { information of } \\
\text { Cash in hand and } \\
\text { Bank balance } \\
\text { will increase.] }\end{array}$} & \multirow[t]{2}{*}{ Manufacturer } & $\begin{array}{l}\text { Service } \\
\text { Provider }\end{array}$ & -.513 & .256 & .117 & -1.12 & .10 \\
\hline & & Trader & .095 & .291 & .943 & -.60 & .79 \\
\hline & \multirow{2}{*}{$\begin{array}{l}\text { Service } \\
\text { Provider }\end{array}$} & Manufacturer & .513 & .256 & .117 & -.10 & 1.12 \\
\hline & & Trader & .608 & .256 & .051 & .00 & 1.22 \\
\hline & \multirow[t]{2}{*}{ Trader } & Manufacturer & -.095 & .291 & .943 & -.79 & .60 \\
\hline & & $\begin{array}{l}\text { Service } \\
\text { Provider }\end{array}$ & -.608 & .256 & .051 & -1.22 & .00 \\
\hline \multirow{6}{*}{$\begin{array}{l}\text { [Proposed One } \\
\text { page Income Tax } \\
\text { Return Form for } \\
\text { having income } \\
\text { less than } 5 \text { lakh } \\
\text { in previous year } \\
\text { will make the } \\
\text { system easy for } \\
\text { disclosure.] }\end{array}$} & \multirow[t]{2}{*}{ Manufacturer } & $\begin{array}{l}\text { Service } \\
\text { Provider }\end{array}$ & -.403 & .260 & .273 & -1.02 & .22 \\
\hline & & Trader & -.190 & 296 & .797 & -.90 & .52 \\
\hline & \multirow{2}{*}{$\begin{array}{l}\text { Service } \\
\text { Provider }\end{array}$} & Manufacturer & .403 & .260 & .273 & -.22 & 1.02 \\
\hline & & Trader & .212 & .260 & .693 & -.41 & .83 \\
\hline & \multirow[t]{2}{*}{ Trader } & Manufacturer & .190 & .296 & .797 & -.52 & .90 \\
\hline & & $\begin{array}{l}\text { Service } \\
\text { Provider }\end{array}$ & -.212 & .260 & .693 & -.83 & .41 \\
\hline \multirow{6}{*}{\begin{tabular}{l}
\multicolumn{3}{l}{ [Presumptive } \\
Tax for small \\
traders now $6 \%$ \\
instead of $8 \%$ for \\
full 1ncash \\
tur1ver.this will \\
increase tax \\
disclosure of \\
small traders.]
\end{tabular}} & \multirow[t]{2}{*}{ Manufacturer } & $\begin{array}{l}\text { Service } \\
\text { Provider }\end{array}$ & -.136 & .247 & .847 & -.73 & .45 \\
\hline & & Trader & -.088 & .285 & .949 & -.77 & .59 \\
\hline & \multirow{2}{*}{$\begin{array}{l}\text { Service } \\
\text { Provider }\end{array}$} & Manufacturer & .136 & .247 & .847 & -.45 & .73 \\
\hline & & Trader & .047 & .251 & .980 & -.55 & .65 \\
\hline & \multirow[t]{2}{*}{ Trader } & Manufacturer & .088 & .285 & .949 & -.59 & .77 \\
\hline & & $\begin{array}{l}\text { Service } \\
\text { Provider }\end{array}$ & -.047 & .251 & .980 & -.65 & .55 \\
\hline \multirow{6}{*}{$\begin{array}{l}\text { [In family, now } \\
\text { more adult group } \\
\text { will indulge in } \\
\text { filing Income } \\
\text { Tax Return after } \\
\text { demonetization } \\
\text { as compared to }\end{array}$} & \multirow[t]{2}{*}{ Manufacturer } & $\begin{array}{l}\text { Service } \\
\text { Provider }\end{array}$ & -.256 & .255 & .577 & -.87 & .35 \\
\hline & & Trader & .000 & .291 & 1.000 & -.70 & .70 \\
\hline & Service & Manufacturer & .256 & .255 & .577 & -.35 & .87 \\
\hline & Provider & Trader & .256 & .255 & .577 & -.35 & .87 \\
\hline & Trader & Manufacturer & .000 & 291 & 1.000 & -.70 & .70 \\
\hline & & Service & -.256 & .255 & .577 & -.87 & .35 \\
\hline
\end{tabular}




\begin{tabular}{|c|c|c|c|c|c|c|c|}
\hline before. ] & & Provider & & & & & \\
\hline \multirow{6}{*}{$\begin{array}{l}\text { [People will } \\
\text { make use of } \\
\text { HUF after } \\
\text { demonetization } \\
\text { which will lead } \\
\text { to more tax } \\
\text { disclosure.] }\end{array}$} & \multirow[t]{2}{*}{ Manufacturer } & $\begin{array}{l}\text { Service } \\
\text { Provider }\end{array}$ & -.308 & .230 & .379 & -.86 & .24 \\
\hline & & Trader & .000 & .262 & 1.000 & -.63 & .63 \\
\hline & \multirow{2}{*}{$\begin{array}{l}\text { Service } \\
\text { Provider }\end{array}$} & Manufacturer & .308 & .230 & .379 & -.24 & .86 \\
\hline & & Trader & .308 & .230 & .379 & -.24 & .86 \\
\hline & \multirow[t]{2}{*}{ Trader } & Manufacturer & .000 & .262 & 1.000 & -.63 & .63 \\
\hline & & $\begin{array}{l}\text { Service } \\
\text { Provider }\end{array}$ & -.308 & .230 & .379 & -.86 & .24 \\
\hline
\end{tabular}

From this descriptive statistics:-

If economy goes cashless, rent received through3 accounts will be disclosed in Income Tax Return. Significance value after applying ANOVA is 0.04 which is below 0.05 , this shows that there is a significant difference in the opinion of the responsers.It can conclude from TUKEY HSD that trader having significant difference of opinion with service provider (its significant level is 0.03 )

Filling of tax return and understanding it is difficult. Significant value after applying ANOVA is 0.092 which is above 0.005 which shows that there is no significance difference in the mean value of their responsers' opinion. In multiple comparisons through TUKEY HSD also among manufacturers, traders and service provider, all are having significant level above 0.05 which shows the same result that there is no significance difference of opinion among them.

Do you have your business registered in any applicable law? Significance level after applying ANOVA is 0.053 which shows that there lies significant difference in it. It is concluded through TUKEY HSD difference lie due to manufacturer with service provider.

Do you disclose your all sources of income? In ANOVA, its significance level is 0.494 which is above 0.05 , there lie no significant mean difference of opinion of the responders' and same result being showed through TUKEY HSD all having significance level of more than 0.05 with each other.

Do you have PAN? Significance level after applying ANOVA is 0.759 which is much above 0.05 , this mean there lie no significant statistical mean difference among it and same through TUKEY HSD, all are having Significance value of more than 0.05 with each other.

Do you file your income tax return on regular basis? Significance level after applying ANOVA is 0.787 which is above 0.05 , this shows there is no significant statistical mean difference among it, almost everyone file their income tax return on regular basis.

Filling of return by CA, CS, other professional person is necessary Significance level after applying ANOVA is 0.577 which is above 0.05 , this shows there is no significance statistical mean difference among it and through TUKEY HSD also, all are having significance value of more than 0.05 .

Do you disclose actual cash in hand and bank balance in income tax return? Significance level after applying ANOVA is 0.022 which is below 0.05 which shows that there is significant mean difference in opinion of respondents. Through TUKEY HSD, there is significance difference of opinion of service provider with trader as they are having significance value of 0.026 which is less than 0.05 , and also manufacture with trader significance level is 0.061 which is above 0.05 but not much higher.

Do you file ITR4? Significance level after applying ANOVA is 0.111 which is above 0.05 , which means that there is no significant statistical mean difference in it. Through TUKEY HSD, there is no significant difference among them as all are having significance value of more than 0.05 .

Do you avail any scheme of undisclosed income issued by the government? Significance level after applying ANOVA is 0.789 which is above 0.05 , which shows there is no significance statistical mean difference among them. 
Taxes have not been paid will now be recovered due to demonetization .Significance level after applying ANOVA is 0.175 , which is above 0.05 , there is no significant statistical mean difference among them, through TUKEY HSD, there is huge significance level of difference between trader with manufacturer and service provider.

Due to demonetization, inflation rate of economy decreases .Significance level after applying ANOVA is 0.215 which shows that there is no significant statistical mean difference among it. Through TUKEY HSD, there is huge significance level of difference between service provider with manufacturer and trader.

People who were holding black money in cash has not been able to exchange much as they would be in a fear of getting prosecuted by the authorities . Its significance level after applying ANOVA is 0.052 which is equal to 0.05 , there lie significance mean difference in opinion of respondents and through TUKEY HSD, it is concluded that difference in opinion lies among service provider with trader.

Increase in return income of persons compared to pre- demonetized return income. Its significance level after applying ANOVA is 0.196 which shows there is no significance statistical mean Difference among it.

Disclosure of all business transactions will increase lead to fair return after demonetization. Its significant level after applying ANOVA is 0.002 which is below 0.05 , which shows there is significant mean difference in opinion of respondents. Through applying TUKEY HSD, it is concluded that the difference of opinion lies in service provider with manufacturer and trader as service provider having significance value of less than 0.05 with both.

Demonetization drive is ago development for payment banks will lead to cashless economy.Its significance level after applying ANOVA is 0.000 which shows there is highly significant difference in opinion of respondents. Through applying TUKEY HSD, it is concluded that difference in opinion lies between traders with service provider as they are having significance level of 0.000 .

Complete disclosure of balances and account details can reduce complication of economy: Its significance level after applying ANOVA is 0.137 which is above 0.05 that means there is no statistical mean significance difference in opinion among them.

No transaction above 3 lakh will be allowed in cash will lead to more transparent income disclosure. Its significance level after applying ANOVA is 0.105 which is above 0.05 which means there is no statistical significance mean difference among them.

After demonetization drive, disclosure of correct and complete information of cash in hand and bank balance will increase. Its significance level in ANOVA is 0.032 , which is below 0.05 shows there is significance difference in opinion of respondents. Through TUKEY HSD, we can say the difference of opinion lie on service provider with trader as their significance level is 0.05 .

One page Income Tax Return form for having income less than 5 lakh in previous year will make the system easy for disclosure .Significance level of it after applying ANOVA is 0.294 which shows there is no statistical mean difference in opinion among them.

Presumptive tax for small traders now $6 \%$ instead of $8 \%$ for full non cash turnover will increase tax disclosure for small traders. Significance level of it after applying ANOVA is 0.861 which is much higher than 0.05.It implies there is no statistical mean difference of opinion among them. All are having almost same views in this context.

In family, now more adult group will indulge in filling income tax return after demonetization as compared to before. Significance level of it after applying ANOVA is 0.477 which is much higher than 0.05 , which implies that there is no statistical mean difference in opinion among them. Through TUKEY HSD, there is high significance level of manufacturer with traders as compared with all.

People will make use of HUF after demonetization which will lead to more tax disclosure. Significance level of it after applying ANOVA is 0.271 which is more than 0.05 , this shows that there is no significant statistical mean difference lie among and through TUKEY HSD also, manufacturer with trader having significance level of 1 , which means they have same views in this context, there is no difference among these two groups and service provider with manufacturer and trader having same significance level, that means there is no difference among them. 


\section{Conclusion:-}

From the Data, the perception of the general public towards tax disclosure after demonetization concluded in the following way. Clusters of manufacturer, trader and service provider shows that they have almost neutral perception in all the questions being asked.

In some questions also, there is significance mean difference of opinion among respondents

1. If economy goes cashless, rent received through accounts will be disclosed in income tax return(service provider and trader)

2. Disclosure of actual cash in hand an bank balance in income tax return.(trader and service provider)

3. Disclosure of all business transactions will increase lead to fair return after demonetization.( service provider with manufacturer and trader)

4. Demonetization drive is a good development for payment banks which will lead to cashless economy(service provider and trader)

5. After demonetization, disclosure of correct and complete information of cash in hand and bank balance will increase. .(service provider and trader)

So, the perception of all the clusters were showing this result that almost they are not sure of tax disclosure after demonetization and on some parts, they are having difference of opinion also. May be the reason for it was it never achieved success before also as it is not a first move ,its being failed in other countries .so public are not very much sure about it so they are neither disagree or agree on it

Trader disagree on Filling of Tax Return and Understanding it is Difficult and inflation rate decreases due to demonetization and people who were holding black money in cash has not been able to exchange much as they would be in a fear of getting prosecuted by the authorities, not all traders disclose actual cash in hand and bank balance in ITR and file ITR4

Manufacturer also disagree on Filling of Tax Return and Understanding it is Difficult Service provider agreed on demonetization drive is ago development for payment banks will lead to cashless economy. Not all Service providers have their business registered in applicable law and file ITR4.

In all questions, almost all responses were of neutral may be the reason for it was it was never achieved success before also as it is not a first move, its being failed in other countries so public are not very much sure about it so they are neither disagree or agree on it.

\section{References:-}

1. Demonetizationhttp://www.investopedia.com/terms/d/demonetization.asp\#ixzz4XuN0GD9o

2. https://pen2print.org/index.php/ijr/article/view/6214/6002

3. https://papers.ssrn.com/sol3/papers.cfm?abstract_id=2933840

4. https://www.researchgate.net/publication/281667605_Tax_compliance_and_the_public_disclosure_of_tax_infor mation_An_AustraliaNorway_comparison. 